\title{
Application of the 2012 revised diagnostic definitions for paediatric multiple sclerosis and immune-mediated central nervous system demyelination disorders
}

\author{
E Daniëlle van Pelt, ${ }^{1}$ Rinze F Neuteboom, ${ }_{1}{ }^{1}$ Immy A Ketelslegers, ${ }^{1}$ Maartje Boon, ${ }^{2}$ \\ Coriene E Catsman-Berrevoets, ${ }^{1}$ Rogier Q Hintzen, ${ }^{1}$ On behalf of the Dutch Study \\ Group for Paediatric MS
}

\begin{abstract}
${ }^{1}$ Department of Neurology, Erasmus MC, Rotterdam, The Netherlands ${ }^{2}$ Department of Neurology, University Medical Centre Groningen, Groningen, The Netherlands
\end{abstract}

\section{Correspondence to} RQ Hintzen, Department of Neurology, Erasmus MC, Room Ee2230, PO Box 2040, Rotterdam 3000 CA, The Netherlands; r.hintzen@erasmusmc.nl

Received 23 September 2013 Revised 11 November 2013 Accepted 14 November 2013 Published Online First 5 December 2013

\section{CrossMark}

\footnotetext{
To cite: van Pelt ED, Neuteboom RF, Ketelslegers IA, et al. J Neurol Neurosurg Psychiatry 2014;85:790-794.
}

\begin{abstract}
Background Recently, the International Paediatric Multiple Sclerosis Study Group (IPMSSG) definitions for the diagnosis of immune-mediated acquired demyelinating syndromes (ADS) of the central nervous system, including paediatric multiple sclerosis (MS), have been revised.

Objective To evaluate the 2012 revised IPMSSG consensus definitions in a cohort of children with ADS prospectively followed from January 2007.

Methods Children with ADS who had an MRI scan obtained within 90 days after first disease onset were included. The sensitivity and specificity of the 2007 and 2012 IPMSSG consensus definitions were assessed. The time to MS diagnosis applying the 2007 and 2012 definitions was compared using survival analysis and log-rank test.
\end{abstract}

Results 82 children with ADS were included. 35 children were diagnosed with paediatric MS, of whom 30 experienced a second clinical event. The final diagnosis corresponded applying either the 2007 or 2012 IPMSSG definitions. The revised 2012 definitions had sufficient sensitivity $(80 \%)$ and high specificity (100\%). MS diagnosis was made 3.4 months earlier $\left(\chi^{2}=8.24\right.$, $\mathrm{p}=0.004$ ) applying the new definitions. In 14 children, MS diagnosis was made at first MRI.

Conclusions MS diagnosis can be made reliable and early using the 2012 IPMSSG consensus definitions. This is beneficial for adequate counselling of children and their families and for early treatment possibilities.

\section{INTRODUCTION}

Recently, the diagnostic criteria for the diagnosis of immune-mediated acquired demyelinating syndromes (ADS) of the central nervous system (CNS), including paediatric multiple sclerosis (MS), have been revised by the International Paediatric Multiple Sclerosis Study Group (IPMSSG). ${ }^{1}$ The new definitions aim to improve consistency in terminology in the heterogeneous group of paediatric demyelinating disorders. They incorporate the 2010 revised McDonald criteria for MS. ${ }^{2}$ Diagnosing MS earlier in the disease course is favourable because disease-modifying treatment (DMT) is also beneficial in children with MS. ${ }^{3}$ Previous studies already showed that the 2010 McDonald MRI criteria, which allow diagnosis at disease onset, are useful for early MS diagnosis in children. ${ }^{4-8}$ But they recommended to be cautious when applying these MRI criteria to young children with acute disseminated encephalomyelitis (ADEM). ${ }^{4}{ }^{6}$ Therefore, the IPMSSG included in the revised 2012 definitions that the $2010 \mathrm{McD}$ onald MRI criteria for dissemination in time (DIT) and dissemination in space (DIS) cannot be applied at first event in children with ADEM and children younger than 12 years old. ${ }^{1}$ In the new IPMSSG consensus definitions, diagnostic criteria for ADEM and neuromyelitis optica (NMO) have been sharpened and the term recurrent ADEM is excluded. Our aim was to evaluate the new 2012 IPMSSG consensus definitions in our clinical cohort of prospectively included children with ADS.

\section{METHODS}

Patients and definitions

Children younger than 18 years, who presented with a first episode of acquired demyelination of the CNS between January 2007 and April 2013, were prospectively included. All children were identified by the Dutch Study Group for Paediatric MS, which consists of paediatric neurologists in 13 major paediatric neurology centres in the Netherlands, or the children were identified by Dutch paediatricians who participate in the NSCK (Netherlands Paediatric Surveillance Unit) as has been described elsewhere. ${ }^{9}$ Children were eligible for this study when a cerebral MRI scan had been obtained within 90 days after first onset of symptoms. All patients had a minimum follow-up time of 2 years, unless they were diagnosed with definite MS. MS diagnosis could be made either on evidence of a second clinical attack at least 30 days after the initial attack or on MRI evidence of DIT within 2 years. Patients were classified as MS, ADEM, clinically isolated syndrome, NMO or other relapsing demyelinating disorders according to the $2007^{10}$ and new $2012^{1}$ IPMSSG consensus definitions.

\section{Standard protocol approvals, registrations and patient consents}

This study was approved by the medical ethical committees of the Erasmus MC in Rotterdam and of the other participating centres. Written informed 
consent was obtained from all children and/or their parents.

\section{MRI analysis}

MRI scans were performed on 1.5-Tesla MRI scanners with slice thicknesses of 3-5 mm. Scans were archived as electronic images. The presence of lesions was determined on T2-weighted and fluid attenuated inversion recovery images. All scans were scored blinded to clinical data by two experienced raters (EDvP and RFN). MRI scans were classified as meeting the 2007 IPMSSG definitions for $\mathrm{MS}^{10}$ (based on the $2001 \mathrm{McD}$ onald MRI criteria ${ }^{11}$ ) or the 2012 IPMSSG definitions for MS $^{1}$ (based on the 2010 McDonald MRI criteria ${ }^{2}$ ). It should be noted that according to the 2007 IPMSSG consensus definitions, DIS can also be met by the combination of abnormal cerebrospinal fluid (CSF) and two lesions on MRI of which one must be in the brain. The 2012 IPMSSG definitions did not incorporate CSF findings anymore. According to the 2007 IPMSSG consensus definitions, MRI criteria for DIT can only be applied on a MRI scan made 3 months after disease onset in children $\geq 10$ years old. ${ }^{10}$ In contrast, using the 2012 IPMSSG consensus definitions MS can be diagnosed after a single event in children $\geq 12$ years old, not meeting ADEM criteria and meeting the $2010 \mathrm{McD}$ onald MRI criteria for DIS and DIT on the baseline MRI scan. ${ }^{1}$

\section{Statistical analysis}

Analyses were performed using SPSS V.20.0. Patient characteristics were compared using $\chi^{2}$ and Student $t$ tests. Test characteristics of the 2007 and 2012 diagnostic criteria (ie, sensitivity, specificity, positive predictive value (PPV), negative predictive value (NPV) and accuracy) were calculated using clinical definite multiple sclerosis (CDMS) as endpoint. Children with MS diagnosis based on MRI criteria who started DMT before CDMS diagnosis $(n=2)$ and those with a follow-up of $<1$ years $(n=3)$ were excluded from the analysis of test characteristics. KaplanMeier survival analyses were used to analyse time to diagnosis according to the 2007 and 2012 IPMSSG consensus definitions. The time to diagnosis was compared using a log-rank test. Survival analysis included all patients with a first MRI with gadolinium and either the presence of a follow-up scan or a diagnosis of CDMS according to the 2010 McDonald MRI criteria.

\section{RESULTS}

Ninety-two children presented with a first demyelinating event between January 2007 and April 2013 and were eligible for this study. Eight patients were excluded because cerebral MRI was not acquired at onset $(n=6$ not at onset, $n=2$ only spinal cord MRI). One patient was excluded because the MRI was not of sufficient quality. One patient with monophasic ADS was excluded because of loss to follow-up within 2 years after onset. Eighty-two children were analysed of whom 41 were younger than 12 years at onset.

In table 1, patient characteristics, including sex, age at first onset of symptoms, type of onset, follow-up time in months and final diagnosis, are presented. CSF data are also presented in table 1 , since the 2007 IPMSSG definitions incorporate CSF in the criteria for DIS, whereas CSF positivity is defined by either the presence of oligloclonal bands or an elevated IgG index. ${ }^{10}$

There were no discrepancies in final diagnosis according to the 2007 or 2012 IPMSSG criteria for paediatric MS and other CNS demyelinating disorders. Thirty-five children are diagnosed with MS according to both 2007 and 2012 IPMSSG definitions. Five of them did not have a second clinical event confirming
CDMS during current follow-up. Two of these patients have started DMT, which may have suppressed a second clinical event. Children with MS were older $(\mathrm{F}=18.35, \mathrm{p}<0.001)$ and have a different type of onset $\left(\chi^{2}=27.6, p<0.001\right)$ as compared with children with monophasic or other relapsing demyelinating disorders. None of the children currently diagnosed with MS presented with ADEM, as defined by a polyfocal event with encephalopathy. ${ }^{1}$

Four patients were diagnosed with $\mathrm{NMO}$ according to both 2007 and 2012 consensus definitions. ${ }^{1}{ }^{10}$ Three of them suffered from a relapsing disease course. Anti-aquaporin- 4 antibodies were tested in 39 patients (48\%) as part of their diagnostic follow-up using a cell-based assay and fluorescence-activated cell sorter. ${ }^{12}$ Two patients had anti-aquaporin-4 IgG seropositivity, one patient with recurrent $\mathrm{NMO}$ and one patient who presented with a brainstem syndrome and was diagnosed with NMO-related spectrum disorder. ${ }^{13}$ Two patients had a final diagnosis of other relapsing demyelinating disorders: one patient suffered from relapsing optic neuritis $(\mathrm{ON})$ in the absence of MRI lesions fulfilling DIT for MS diagnosis and aquaporin-4 antibodies for NMO spectrum diagnosis. The other patient suffered from a first event of ADEM followed by one event of ON without the presence of new MRI lesions during follow-up. ${ }^{14}$ None of the children with ADEM onset had a subsequent event of ADEM (multiphasic ADEM).

In table 2, sensitivity and specificity of the 2007 and 2012 IPMSSG consensus definitions are presented with CDMS as endpoint using only the baseline characteristics and using also follow-up MRI.

The 2012 IPMSSG consensus definitions had better sensitivity (79\% vs 68\%), NPV (88\% vs 71\%) and accuracy (92\% vs $82 \%$ )

Table 1 Patient characteristics

\begin{tabular}{|c|c|c|c|c|c|}
\hline & \multicolumn{2}{|c|}{$\begin{array}{l}\text { Multiple } \\
\text { sclerosis }(n=35)\end{array}$} & \multicolumn{2}{|l|}{$\begin{array}{l}\text { Other ADS } \\
(n=47)\end{array}$} & $p$ Value \\
\hline Women/men & $20 / 15$ & & $30 / 17$ & & NS \\
\hline \multicolumn{6}{|l|}{ Age at onset } \\
\hline Mean, SD & $14.7 \pm 2.8$ & & $7.5 \pm 4.8$ & & $p<0.001$ \\
\hline Range & $5.3-17.7$ & & $1.0-16.7$ & & \\
\hline Median & 15.5 & & 6.2 & & \\
\hline \multicolumn{3}{|l|}{ Type of onset (\%) } & & & $p<0.001$ \\
\hline Optic neuritis & \multicolumn{2}{|l|}{$6(17.1 \%)$} & \multicolumn{2}{|l|}{10 (21.3\%) } & \\
\hline Transverse myelitis & \multicolumn{2}{|l|}{$1(2.9 \%)$} & \multicolumn{2}{|l|}{$2(4.3 \%)$} & \\
\hline Other monofocal CIS & \multicolumn{2}{|l|}{$12(34.3 \%)$} & \multicolumn{2}{|l|}{$2(4.3 \%)$} & \\
\hline Polyfocal CIS & \multicolumn{2}{|l|}{$16(45.7 \%)$} & \multicolumn{2}{|l|}{$13(27.7 \%)$} & \\
\hline ADEM & \multicolumn{2}{|l|}{-} & \multicolumn{2}{|l|}{$20(42.6 \%)$} & \\
\hline CSF analysis performed (\%) & \multicolumn{2}{|l|}{$31(88.6 \%)$} & \multicolumn{2}{|l|}{$45(95.7 \%)$} & NS \\
\hline Positive CSF (valid \%)* & \multicolumn{2}{|l|}{$26(83.9 \%)$} & \multicolumn{2}{|l|}{$8(17.8 \%)$} & $\mathrm{p}<0.001$ \\
\hline $\begin{array}{l}\text { Follow-up time, months } \\
\text { (mean, SD) }\end{array}$ & \multicolumn{2}{|l|}{$32 \pm 17$} & \multicolumn{2}{|l|}{$38 \pm 12$} & NS \\
\hline \multirow{4}{*}{$\begin{array}{l}\text { Final diagnosis according } \\
\text { to the } 2007 \text { and } 2012 \\
\text { consensus definitions }\end{array}$} & CDMS & 30 & $\mathrm{CIS}$ & 22 & \\
\hline & $\begin{array}{l}\text { Definite MS } \\
\text { based on }\end{array}$ & 5 & ADEM & 19 & \\
\hline & MRI & & NMO & 4 & \\
\hline & \multicolumn{2}{|l|}{ evidence } & $\begin{array}{l}\text { Other } \\
\text { relapsing } \\
\text { ADS }\end{array}$ & 2 & \\
\hline
\end{tabular}


Table 2 Test characteristics of the MRI criteria include the 2007 and 2012 IPMSSG consensus definitions when applied to distinguish MS from other acquired demyelinating syndromes (ADS) of the CNS

\begin{tabular}{|c|c|c|c|c|c|c|c|}
\hline & $\begin{array}{l}\text { First MRI } \\
2007 \text { DIS }\end{array}$ & $\begin{array}{l}\text { First MRI } \\
2007 \text { DIS }\end{array}$ & $\begin{array}{l}\text { First MRI } \\
2012 \text { DIS }\end{array}$ & $\begin{array}{l}\text { First MRI } \\
2012 \text { DIT }\end{array}$ & $\begin{array}{l}\text { First MRI } \\
2012 \text { DIS+DIT }\end{array}$ & $\begin{array}{l}\text { First and second MRI } \\
2007 \text { DIS+DIT }\end{array}$ & $\begin{array}{l}\text { First and second MRI } \\
2012 \text { DIS+DIT }\end{array}$ \\
\hline & 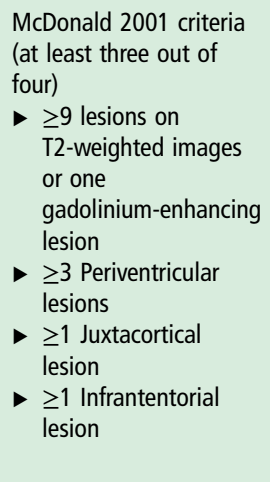 & $\begin{array}{l}\text { Abnormal CSF } \\
\text { Two lesions on the } \\
\text { MRI of which one } \\
\text { must be in the brain* }\end{array}$ & $\begin{array}{l}\text { McDonald } 2010 \text { criteria } \\
\text { (at least two out of four) } \\
-\geq 1 \text { Spinal cord } \\
-\geq 1 \text { Periventricular } \\
-\geq 1 \text { Juxtacortical } \\
\text { lesion } \\
-\geq 1 \text { Infrantentorial } \\
\text { lesion }\end{array}$ & $\begin{array}{l}\text { McDonald } 2010 \text { criteria } \\
\text { - Simultaneous } \\
\text { presence of } \\
\text { asymptomatic } \\
\text { gadolinium-enhancing } \\
\text { and non-enhancing } \\
\text { lesions } \\
\text { Excluding children } \\
\leq 12 \text { years oldt }\end{array}$ & $\begin{array}{l}\text { McDonald } 2010 \text { for DIS } \\
\text { and DIT at baseline } \\
\text { Excluding children } \\
\leq 12 \text { years oldt }\end{array}$ & 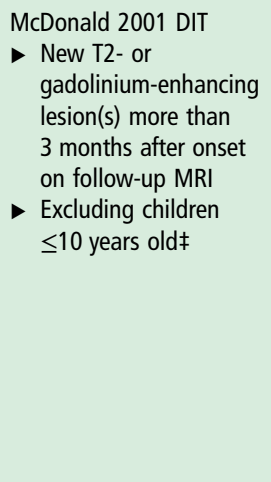 & $\begin{array}{l}\text { McDonald } 2010 \text { DIT } \\
\text { New T2 and/or } \\
\text { gadolinium-enhancing } \\
\text { lesion(s) on follow-up } \\
\text { MRI, with reference } \\
\text { to a baseline scan } \\
\text { irrespective of the } \\
\text { timing of the baseline } \\
\text { scan } \\
\text { Simultaneous } \\
\text { presence of } \\
\text { asymptomatic } \\
\text { gadolinium-enhancing } \\
\text { and non-enhancing } \\
\text { lesions at any time§ }\end{array}$ \\
\hline Sensitivity (\%) & 70 & 75 & 93 & 63 & 58 & 68 & 79 \\
\hline Specificity (\%) & 96 & 89 & 77 & 100 & 100 & 100 & 100 \\
\hline PPV (\%) & 91 & 81 & 71 & 100 & 100 & 100 & 100 \\
\hline NPV (\%) & 83 & 85 & 95 & 82 & 80 & 71 & 88 \\
\hline Accuracy (\%) & 86 & 84 & 83 & 86 & 85 & 82 & 92 \\
\hline
\end{tabular}

In this table, test characteristics are presented with CDMS as endpoint using first and second MRI.

* Calculated for patients when CSF was performed (n=73).

tCalculated for children who had a first MRI with gadolinium $(n=65)$.

$\ddagger$ Calculated for children who had a second MRI following 3 months after onset $(n=39)$.

$\S$ Calculated for children who had their first MRI with gadolinium and/or had second MRI, excluding children $\leq 12$ years old for DIT 2010 at baseline $(n=73)$,

CDMS, clinical definite multiple sclerosis; CNS, central nervous system; CSF, cerebrospinal fluid; DIS, dissemination in space; DIT, dissemination in time; IPMSSG, International Paediatric Multiple Sclerosis Study Group; MS, multiple sclerosis; NPV,

negative predictive value; PPV, positive predictive value. 
compared with the 2007 definitions. The test characteristics differed not significantly. For the calculations of DIS and DIT 2010 McDonald MRI criteria at onset, only children who had a first MRI with gadolinium were included. Two patients who presented with ADEM fulfilled the 2010 McDonald MRI criteria at the first MRI and one of them also fulfilled DIS and DIT 2001 and 2010 McDonald MRI criteria at second MRI (>3 months after onset). Both these patients had negative CSF at onset and did not have a second event. Follow-up time of these patients was 53 and 58 months, respectively. However, according to the 2012 IPMSSG definitions, the 2010 McDonald MRI criteria do not apply for children with an ADEM onset and both children were younger than 12 years old. Therefore, according to both the 2007 and 2012 IPMSSG consensus definitions, these children cannot be diagnosed as MS. In contrast, two other patients presenting with ADS and younger than 12 years fulfilled the 2010 McDonald MRI criteria for DIS and DIT at baseline, but because of their age they were not diagnosed with MS at first MRI according to 2012 IPMSSG consensus definitions. Both these patients eventually showed clinical progression and thus were diagnosed with CDMS and turned out to have a false-negative test result at baseline. Applying the 2010 DIS and DIT McDonald MRI criteria at disease onset, thus irrespective of the age limit of 12 years, resulted in similar test characteristics (sensitivity 67\%, specificity 100\%, PPV 100\%, NPV 83\%) as when applied only in children older than 12 years (table 2, column 5). In a subgroup of 41 children younger than 12 years at onset, including four patients with MS, we found a lower sensitivity (50\%) but higher NPV (95\%) applying the 2010 McDonald MRI criteria, irrespective of the age limit at first MRI, but a high sensitivity $(100 \%)$ when a follow-up MRI is included.

Sixty-five children, including 24 children with MS, had a first MRI with gadolinium administration. MS was diagnosed in 14 $(47 \%)$ children at first MRI (excluding two children younger than 12 years) using the 2012 IPMSSG definitions. Six (20\%) of these patients had a spinal cord lesion contributing to DIS McDonald MRI criteria.

Twenty-seven children were eligible for the survival analysis. Kaplan-Meier survival curves (figure 1) show the time to MS diagnosis using the 2007 versus 2012 IPMSSG consensus definitions. MS diagnosis in children can be made significantly earlier $\left(\chi^{2}=8.24, p=0.004\right)$ using the revised 2012 consensus definitions based on the 2010 McDonald MRI criteria, similar as in adults. ${ }^{15}$ The mean time to diagnosis was $8.5 \pm 7.4$ months and $5.1 \pm 7.2$ months applying the 2007 and 2012 IPMSSG definitions, respectively $(t=6.87, p<0.001)$. The average time to MS diagnosis is 3.4 months earlier using the 2012 IPMSSG definitions. The mean time to a second event defining CDMS was 10.1 \pm 8.7 months.

\section{DISCUSSION}

In this study, the revised 2012 IPMSSG consensus definitions for paediatric MS and immune-mediated CNS demyelinating disorders were evaluated in a prospective nationwide cohort of children aged 1.0-17.7 years old with the full spectrum of immune-mediated CNS demyelination disorders. The 2010 revised McDonald MRI criteria have already been studied and found useful in children with CNS demyelination. ${ }^{4-8}$ In contrast to these previous studies, not only the revised 2010 McDonald MRI criteria were considered in the present study, but also other clinical characteristics as proposed in the 2012 IPMSSG definitions. This means that the 2010 McDonald MRI criteria for DIT on the first MRI can only be applied to children of 12 years and older and having a first clinical event not meeting

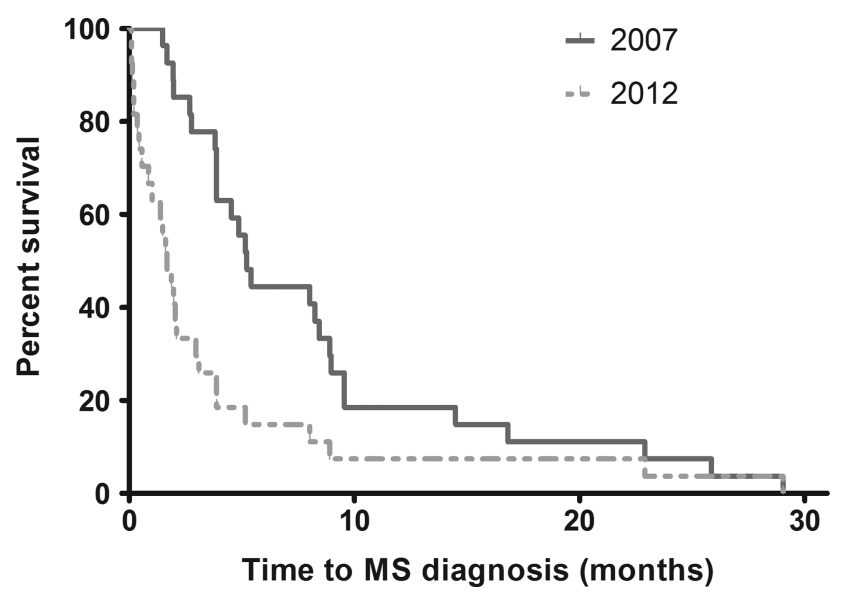

Figure 1 Kaplan-Meier survival curves showing the time to MS diagnosis using the 2007 versus 2012 International Paediatric Multiple Sclerosis Study Group (IPMSSG) consensus definitions. The 2012 IPMSSG definitions allow for an earlier diagnosis $\left(\chi^{2}=8.24, p=0.004\right)$.

criteria for ADEM. This study showed that the 2012 IPMSSG consensus definitions allow for an equally reliable, but earlier MS diagnosis in all children, also those younger than 12 years. Fourteen (47\%) MS patients could be diagnosed at first MRI using the 2012 IPMSSG definitions. Six of these patients (20\%) fulfilled DIS McDonald criteria because of the presence of spinal cord lesion(s), confirming the importance of a spinal cord MRI at onset. ${ }^{7}$

An important finding in our study is that the final diagnosis corresponded applying either the 2007 or 2012 IPMSSG consensus definitions. However, using the 2012 definitions, a diagnosis of MS can be made earlier, which is beneficial for adequate counselling of the children and their families, as prognosis can be uncertain after ADS, and children with MS may benefit from early treatment.

Test characteristics were better when applying the 2012 IPMSSG definitions compared with the 2007 definitions. When including follow-up MRI scans for the application of the 2007 and 2012 IPMSSG definitions, both have a high specificity and PPV, indicating the absence of false-positive test results. This is because the McDonald MRI criteria do not apply for children aged $\geq 10$ years (2007 IPMSSG definitions) and $\geq 12$ years at baseline (2012 IPMSSG definitions) and for children with an onset of ADEM (2012 IPMSSG definitions). Test characteristics for DIT based on MRI criteria according to 2007 IPMSSG definitions were calculated for $39 / 77$ patients with a follow-up scan $\geq 3$ months after the onset of clinical symptoms. This may have introduced some bias as the decision to make a second MRI might not be fully independent of the initial observed disease activity.

When applying the $2010 \mathrm{McD}$ onald MRI criteria to all children (regardless of age), test characteristics are comparable although sensitivity would be higher, due to the finding that two patients $\leq 12$ years old with paediatric MS tested false negative. In a subgroup of children aged younger than 12 years old, we found lower sensitivity and NPV, indicating more falsenegative results. A second MRI increased the sensitivity. When the DIS and DIT 2010 criteria are not applied in children with ADEM at onset but regardless of age, high specificity and PPV were found, which might warrant exclusion of the age limit. Further investigation of the age limit is needed.

In contrast to the study of Sadaka et $a l,{ }^{4}$ we did not find $100 \%$ sensitivity and NPV for the 2010 McDonald MRI 
criteria. This is probably explained by the fact we did not use a standardised MRI protocol, including systematic frequent follow-up MRIs. Despite the lack of a systematic MRI protocol, we did find earlier MS diagnosis in the Dutch cohort which resembles general clinical practice.

We only included patients with a minimum follow-up of 2 years (unless they were diagnosed with MS based on MRI criteria within 2 years $(n=3)$ ) because the time interval between first and second attack in paediatric MS is typically less than 12 months. ${ }^{16}{ }^{17}$ Despite a relatively long follow-up, it still might be that patients currently defined as monophasic will experience relapses in future.

A first onset of ADEM is an important clinical parameter when using the 2012 consensus definitions. In our prospective cohort, none of the patients with a first onset of ADEM developed MS during follow-up, confirming its role as a negative predictor for MS. Using the 2012 consensus definitions, children with ADEM can be diagnosed with MS earlier if they experience a second non-ADEM event with new MRI lesions at least 3 months after onset. We did not have such patients in our current cohort. Relapse after ADEM typically occurs within 2 years after onset, but in a small subgroup can occur many years later. $^{1}$

Using the new 2012 consensus definitions for the diagnosis of paediatric MS, CSF analysis is not strictly needed. Although in clinical practice CSF analysis in children presenting with neurological symptoms is still of importance in order to exclude other diagnoses as infections and malignancies, ${ }^{18}$ it should be further investigated if CSF analysis is of use in doubtful cases where MRI criteria have not been completely fulfilled.

The results of our prospective multicentre cohort show that the 2012 IPMSSG consensus definitions apply well for a reliable and early diagnosis of paediatric MS and ADS.

\section{Acknowledgements The authors thank all the children and their families who participated in the Dutch Study for Paediatric MS; the members of the Dutch Study Group for Paediatric MS: KGJ van Dijk, Department of Paediatrics, Rijnstate Hospital, Arnhem; RJ Vermeulen, Department of Child Neurology, VU Medical Centre Amsterdam; KPJ Braun, Department of Paediatric Neurology, University Medical Centre Utrecht, Utrecht; EH Niks and CMPCD Peeters-Scholte, Department of Neurology, Leiden University Medical Centre, Leiden; WCG Overweg-Plandsoen and BT Poll, The Department of Paediatric Neurology, Emma Children's Hospital/AMC, Amsterdam; EAJ Peeters and IN Snoeck, Department of Neuropaediatrics, Haga Hospital, The Hague; JF de Rijk- van Andel, Department of Neurology, Amphia Hospital, Breda; JPA Samijn, Department of Neurology, Maasstad Hospital, Rotterdam; H Stroink and A Verrips, Department of Neurology, Canisius-Wilhelmina Hospital, Nijmegen; JSH Vles, Department of Neurology, University Hospital Maastricht, Maastricht; MAAP Willemsen, Department of Paediatric Neurology, Radboud University Nijmegen Medical Centre, Nijmegen.}

Funding This study was supported by the Dutch MS Research Foundation.

Contributors The work presented here was carried out in collaboration between all authors. IAK and EDvP were responsible for data collection. EDvP and RFN performed the data analysis. EDvP, RFN, IAK and RQH interpreted the results and wrote the paper. All authors have contributed to the manuscript.

\section{Competing interests None.}

Ethics approval Medical ethical committees of the Erasmus MC in Rotterdam and participating centres in the Dutch Study Group for Paediatric MS.

Provenance and peer review Not commissioned; externally peer reviewed.

\section{REFERENCES}

1 Krupp LB, Tardieu M, Amato MP, et al. International Pediatric Multiple Sclerosis Study Group criteria for pediatric multiple sclerosis and immune-mediated central nervous system demyelinating disorders: revisions to the 2007 definitions. Mult Scler 2013;19:1261-67.

2 Polman $\mathrm{CH}$, Reingold SC, Banwell B, et al. Diagnostic criteria for multiple sclerosis: 2010 revisions to the McDonald criteria. Ann Neurol 2011;69:292-302.

3 Chitnis T, Tenembaum S, Banwell B, et al. Consensus statement: evaluation of new and existing therapeutics for pediatric multiple sclerosis. Mult Scler 2012;18:116-27.

4 Sadaka Y, Verhey LH, Shroff MM, et al. 2010 McDonald criteria for diagnosing pediatric multiple sclerosis. Ann Neurol 2012;72:211-23.

5 Sedani $S$, Lim MJ, Hemingway $C$, et al. Paediatric multiple sclerosis: examining utility of the McDonald 2010 criteria. Mult Scler 2012;18:679-82.

6 Tantsis EM, Prelog K, Brilot F, et al. Risk of multiple sclerosis after a first demyelinating syndrome in an Australian Paediatric cohort: clinical, radiological features and application of the McDonald 2010 MRI criteria. Mult Scler 2013;19:1749-59.

7 Hummel HM, Bruck W, Dreha-Kulaczewski S, et al. Pediatric onset multiple sclerosis: McDonald criteria 2010 and the contribution of spinal cord MRI. Mult Scler 2013;19:1330-35

8 Kornek B, Schmitl B, Vass K, et al. Evaluation of the 2010 McDonald multiple sclerosis criteria in children with a clinically isolated syndrome. Mult Scler 2012;18:1768-74.

9 Ketelslegers IA, Catsman-Berrevoets CE, Neuteboom RF, et al. Incidence of acquired demyelinating syndromes of the CNS in Dutch children: a nationwide study. J Neurol 2012;259:1929-35.

10 Krupp LB, Banwell B, Tenembaum S, et al. Consensus definitions proposed for pediatric multiple sclerosis and related disorders. Neurology 2007;68:S7-12.

11 McDonald WI, Compston A, Edan G, et al. Recommended diagnostic criteria for multiple sclerosis: guidelines from the International Panel on the diagnosis of multiple sclerosis. Ann Neurol 2001;50:121-27.

12 Ketelslegers IA, Modderman PW, Vennegoor A, et al. Antibodies against aquaporin-4 in neuromyelitis optica: distinction between recurrent and monophasic patients. Mult Scler 2011;17:1527-30.

13 Wingerchuk DM, Lennon VA, Lucchinetti CF, et al. The spectrum of neuromyelitis optica. Lancet Neurol 2007;6:805-15.

14 Huppke P, Rostasy K, Karenfort M, et al. Acute disseminated encephalomyelitis followed by recurrent or monophasic optic neuritis in pediatric patients. Mult Scler 2013;19:941-46

15 Runia TF, Jafari N, Hintzen RQ. Application of the 2010 revised criteria for the diagnosis of multiple sclerosis to patients with clinically isolated syndromes. Eur J Neurol 2013;20:1510-16.

16 Banwell B, Bar-Or A, Arnold DL, et al. Clinical, environmental, and genetic determinants of multiple sclerosis in children with acute demyelination: a prospective national cohort study. Lancet Neurol 2011;10:436-45.

17 Mikaeloff $Y$, Suissa S, Vallee L, et al. First episode of acute CNS inflammatory demyelination in childhood: prognostic factors for multiple sclerosis and disability. J Pediatr 2004;144:246-52.

18 Banwell B, Ghezzi A, Bar-Or A, et al. Multiple sclerosis in children: clinical diagnosis, therapeutic strategies and future directions. Lancet Neurol 2007;6:887-902. 